\title{
Planetary Nebulae in Elliptical Galaxies: Dynamical Models for Centaurus A
}

\section{Anne Mathieu and Herwig Dejonghe}

Sterrenkundig Observatorium, University of Ghent, Belgium

We use planetary nebulae major- and minor-axis kinematics (Hui et al. 1995) of the dust-lane elliptical galaxy NGC 5128 (Centaurus A) to build triaxial dynamical models.

Centaurus $\mathrm{A}$ is a round galaxy, so as a first approximation we assume a spherical gravitational potential. The data consist in the flattened photometry of this E2 galaxy (well fitted by a de Vaucouleurs profile along the major axis) and the observed kinematics of Cen A (rotation curves and velocity dispersion profiles along the major and minor axes). The modeling technique is based on a quadratic programming algorithm which allows us to determine a positive distribution function (DF) written as a linear combination of simple basis functions such that the corresponding moments of the DF fit the observations i.e. the photometric and kinematical data. The basis functions are Fricke components (Mathieu et al. 1996a) allowing for rotation about the intrinsic major and minor axes of the galaxy. We show that no self-consistent models can fit the whole set of data, but models with a dark halo give pretty good fits. The total mass range interior to $50 \mathrm{kpc}$ for Cen $\mathrm{A}$ is $3 \times 10^{11} M_{\odot} \leq M_{\text {tot }} \leq 5 \times 10^{11} M_{\odot}$, of which $50 \%$ is dark matter.

In order to get a more accurate picture of the dynamics of Cen $\mathrm{A}$, a triaxial potential is needed. To this end, we have developped a deprojection technique to approximate the gravitational potential of a galaxy by a triaxial Stäckel potential which admits three exact analytical integrals of motion (Mathieu \& Dejonghe 1996b). We will use this potential to build fully triaxial dynamical models with other basis components taking advantage of the Stäckel nature of the potential.

\section{REFERENCES}

Hui, X., Ford, H. C., Freeman, K. C., Dopita, M. A., 1995, Astrophys. Journ., 449, 592.

Mathieu, A., Dejonghe, H., Hui, X., 1996a, Astron. Astrophys., 309, 30.

Mathieu, A., Dejonghe, H., 1996b, Astron. Astrophys., 314, 25. 